\title{
On the Mass Concentration for Bose-Einstein Condensates with Attractive Interactions
}

\author{
Yujin Guo* and Robert Seiringer ${ }^{\dagger}$
}

September 20, 2018

\begin{abstract}
We consider two-dimensional Bose-Einstein condensates with attractive interaction, described by the Gross-Pitaevskii functional. Minimizers of this functional exist only if the interaction strength $a$ satisfies $a<a^{*}=\|Q\|_{2}^{2}$, where $Q$ is the unique positive radial solution of $\Delta u-u+u^{3}=0$ in $\mathbb{R}^{2}$. We present a detailed analysis of the behavior of minimizers as $a$ approaches $a^{*}$, where all the mass concentrates at a global minimum of the trapping potential.
\end{abstract}

Dedicated to Nassif Ghoussoub on the occasion of his $60^{\text {th }}$ birthday.

Keywords: Bose-Einstein condensation; attractive interactions; Gross-Pitaevskii functional; mass concentration; symmetry breaking.

$M S C(2010): 35 Q 40,46 N 50,82 D 50$

\section{Introduction and Main Results}

The phenomenon of Bose-Einstein condensation (BEC) has been investigated intensively since its first realization in cold atomic gases 2, 11. In these experiments, a large number of (bosonic) atoms are confined to a trap and cooled to very low temperature. Condensation of a large fraction of particles into the same one-particle state is observed below a critical temperature.

These Bose-Einstein condensates display various interesting quantum phenomena [10, 9 , 12, 4, such as the appearance of quantized vortices in rotating traps, the effective lower dimensional behavior in strongly elongated traps, etc. The forces between the atoms in the condensates can be either attractive or repulsive. In the attractive case, the system collapses if the particle number increases beyond a critical value; see, e.g., [5, 6, 18, 20, 36]

\footnotetext{
*Wuhan Institute of Physics and Mathematics, Chinese Academy of Sciences, P.O. Box 71010, Wuhan 430071, P. R. China. Email: yjguo@wipm.ac.cn. Y. Guo is partially supported by NSFC grants 11241003 and 11322104 .

${ }^{\dagger}$ IST Austria, Am Campus 1, 3400 Klosterneuburg, Austria. Email: robert.seiringer@ist.ac.at. R. Seiringer is partially supported by the Natural Science and Engineering Research Council of Canada.
} 
or [10, Sec. III.B]. Our main interest in the present paper is to investigate the details of this collapse.

We study Bose-Einstein condensates with attractive interactions in two dimensions, described by the Gross-Pitaevskii (GP) energy functional [15, 16, 34]. In suitable units, the GP functional is given by

$$
E_{a}(u):=\int_{\mathbb{R}^{2}}\left(|\nabla u(x)|^{2}+V(x)|u(x)|^{2}\right) d x-\frac{a}{2} \int_{\mathbb{R}^{2}}|u(x)|^{4} d x, \quad u \in \mathcal{H},
$$

where $a>0$ describes the strength of the attractive interactions, and $\mathcal{H}$ is defined as

$$
\mathcal{H}:=\left\{u \in H^{1}\left(\mathbb{R}^{2}\right): \int_{\mathbb{R}^{2}} V(x)|u(x)|^{2} d x<\infty\right\}
$$

with associated norm $\|u\|_{\mathcal{H}}=\left\{\int_{\mathbb{R}^{2}}\left(|\nabla u|^{2}+[1+V(x)]|u(x)|^{2}\right) d x\right\}^{\frac{1}{2}}$. We note that in the case of repulsive interactions (corresponding to $a<0$ in (1.1)), the GP functional can be rigorously derived from the quantum many-body problem in a suitable low-density limit 28, 29, 30, 27. Its validity in the attractive case remains an open problem, however.

We are interested in minimizers of (1.1) under the unit mass constraint

$$
\int_{\mathbb{R}^{2}}|u(x)|^{2} d x=1
$$

Alternatively, one may want to impose the constraint $\int_{\mathbb{R}^{2}}|u(x)|^{2} d x=N$, with $N$ the particle number, but this latter case can easily be reduced to the previous one, by minimizing under the constraint (1.3) but simply replacing $a$ by $N a$. Hence we prefer to work with (1.3) instead.

We assume that the function $V: \mathbb{R}^{2} \rightarrow \mathbb{R}$ is locally bounded and satisfies $V(x) \rightarrow \infty$ as $|x| \rightarrow \infty$. By adding a suitable constant, we may impose the condition $\inf _{x \in \mathbb{R}^{2}} V(x)=0$ without loss of generality. We define the GP energy to be

$$
e(a):=\inf _{\left\{u \in \mathcal{H},\|u\|_{2}^{2}=1\right\}} E_{a}(u) .
$$

Note that, without loss of generality, we can restrict the minimization to non-negative functions, since $E_{a}(u) \geq E_{a}(|u|)$ for any $u \in \mathcal{H}$. This follows from the fact that $|\nabla| u|| \leq|\nabla u|$ a.e. in $\mathbb{R}^{2}$.

We start by investigating the finiteness of $e(a)$. As an infimum over affine-linear functions, $e(a)$ is concave, and it is clearly also decreasing in $a$. Using the fact that $\int|\nabla u|^{2}$ and $\int|u|^{4}$ behave the same under ( $L^{2}$-preserving) scaling of $u$, it is easy to see that either $e(a) \geq 0$ or $e(a)=-\infty$. Hence a simple variational argument yields the existence of an $a^{*} \geq 0$ such that $e(a)=-\infty$ for $a>a^{*}$. It turns out that the value of $a^{*}$ can be determined by solving the nonlinear scalar field equation

$$
-\Delta u+u-u^{3}=0 \text { in } \mathbb{R}^{2} \text {, where } u \in H^{1}\left(\mathbb{R}^{2}\right) .
$$

It is well-known [14, 25, 32, 23, that, up to translations, (1.5) admits a unique positive solution, which can be taken to be radially symmetric about the origin. We shall denote it by $Q$. Moreover, we recall from 38 the following Gagliardo-Nirenberg inequality

$$
\int_{\mathbb{R}^{2}}|u(x)|^{4} d x \leq \frac{2}{\|Q\|_{2}^{2}} \int_{\mathbb{R}^{2}}|\nabla u(x)|^{2} d x \int_{\mathbb{R}^{2}}|u(x)|^{2} d x, \quad u \in H^{1}\left(\mathbb{R}^{2}\right),
$$


where equality is achieved for $u(x)=Q(x)$. This inequality can be used to obtain the following theorem concerning the existence and non-existence of minimizers for the minimization problem (1.4).

Theorem 1. Let $Q$ be the unique positive radial solution of (1.5). Suppose $V \in L_{\text {loc }}^{\infty}\left(\mathbb{R}^{2}\right)$ satisfies $\lim _{|x| \rightarrow \infty} V(x)=\infty$ and $\inf _{x \in \mathbb{R}^{2}} V(x)=0$. Then

1. If $0 \leq a<a^{*}:=\|Q\|_{2}^{2}$, there exists at least one minimizer for (1.4).

2. If $a \geq a^{*}:=\|Q\|_{2}^{2}$, there is no minimizer for (1.4).

Moreover, $e(a)>0$ for $a<a^{*}, \lim _{a \rightarrow a^{*}} e(a)=e\left(a^{*}\right)=0$, and $e(a)=-\infty$ for $a>a^{*}$.

The proof of the existence of minimizers in the case $0 \leq a<a^{*}$ follows standard arguments (see [35, 40]) and we include it here for completeness. A numerical computation [38] yields $\|Q\|_{2}^{2}=2 \pi \times 1.86225 \cdots$. It is not difficult to obtain analytical bounds as well. We shall demonstrate in Lemma 1 below that

$$
2 \pi \leq\|Q\|_{2}^{2} \leq 2 \pi e \ln 2 \approx 2 \pi \times 1.88417 \cdots .
$$

Note that since the parameter $a$ in (1.1) has to be interpreted as particle number times interaction strength, as discussed after Eq. (1.3) above, the existence of the threshold $a^{*}$ described in Theorem 1 yields the existence of a critical particle number for collapse of the Bose-Einstein condensate [10. Theorem 1 also implies that the trap shape does not affect the critical particle number (compare with 39]).

If $u$ is a non-negative minimizer of (1.1) for $a<a^{*}$, it satisfies the GP equation

$$
-\Delta u(x)+V(x) u(x)=\mu u(x)+a u(x)^{3} \quad \text { in } \quad \mathbb{R}^{2}
$$

for a suitable Lagrange parameter $\mu$. From this equation one can deduce exponential decay of $u$ via standard techniques. Moreover, the maximum principle implies that $u$ is strictly positive, and elliptic regularity yields smoothness properties of $u$ depending on the smoothness assumptions on $V$. These results are standard (see, e.g., [8]) and we shall not investigate the details here.

Our main result concerns the behavior of minimizers $u_{a}$ of (1.1) as a approaches the critical value $a^{*}$ from below. Since $e\left(a^{*}\right)=0$, it is easy to see that $\int_{\mathbb{R}^{2}} V(x)\left|u_{a}(x)\right|^{2} d x \rightarrow$ $0=\inf _{x \in \mathbb{R}^{2}} V(x)$ as $a \rightarrow a^{*}$, hence this behavior depends on the behavior of $V$ near its minima. The functions $u_{a}$ can be expected to concentrate at the flattest minimum of $V$. If $V$ has a unique minimum, $\left|u_{a}(x)\right|^{2}$ converges to a $\delta$-function located at this minimum.

In the following, we shall assume that the trap potential $V$ has $n \geq 1$ isolated minima, and that in their vicinity $V$ behaves like a power of the distance from these points. More precisely, we shall assume that there exist $n \geq 1$ distinct points $x_{i} \in \mathbb{R}^{2}$ with $V\left(x_{i}\right)=0$, while $V(x)>0$ otherwise. Moreover, there are numbers $p_{i}>0$ and a constant $C>0$ such that

$$
V(x)=h(x) \prod_{i=1}^{n}\left|x-x_{i}\right|^{p_{i}} \quad \text { with } C<h(x)<1 / C \text { for all } x \in \mathbb{R}^{2} .
$$

We also need to assume that $\lim _{x \rightarrow x_{i}} h(x)$ exists for all $1 \leq i \leq n$. 
Our method certainly allows to relax these assumptions in various ways. For instance, a very rapid increase at infinity (which could lead to $Q \notin \mathcal{H}$ ) could be handled by introducing suitable additional cut-off functions. For the sake of simplicity we shall not strive to cover the most general class of trap potentials here. We note that new ingredients in the proof are needed to treat the case of radially symmetric, ring-shaped potentials (with a continuum of minima) and the corresponding analysis will be presented elsewhere [17.

Let $p=\max \left\{p_{1}, \ldots, p_{n}\right\}$, and let $\lambda_{i} \in(0, \infty]$ be given by

$$
\lambda_{i}=\left(\frac{p}{2} \int_{\mathbb{R}^{2}}|x|^{p} Q(x)^{2} d x \lim _{x \rightarrow x_{i}} \frac{V(x)}{\left|x-x_{i}\right|^{p}}\right)^{\frac{1}{2+p}} .
$$

Define $\lambda=\min \left\{\lambda_{1}, \cdots, \lambda_{n}\right\}$ and let

$$
\mathcal{Z}:=\left\{x_{i}: \lambda_{i}=\lambda\right\}
$$

denote the locations of the flattest global minima of $V(x)$.

Theorem 2. Suppose $V$ satisfies the assumptions above, and let $u_{a}$ be a non-negative minimizer of (1.1) for $a<a^{*}$. Given a sequence $\left\{a_{k}\right\}$ with $a_{k} \nearrow a^{*}$ as $k \rightarrow \infty$, there exists a subsequence (still denoted by $\left\{a_{k}\right\}$ ) and an $x_{0} \in \mathcal{Z}$ such that

$$
\lim _{k \rightarrow \infty}\left(a^{*}-a_{k}\right)^{\frac{1}{2+p}} u_{a_{k}}\left(x_{0}+x\left(a^{*}-a_{k}\right)^{\frac{1}{2+p}}\right)=\frac{\lambda Q(\lambda x)}{\|Q\|_{2}}
$$

strongly in $L^{q}\left(\mathbb{R}^{2}\right)$ for $2 \leq q<\infty$.

The theorem gives a detailed description of the behavior of GP minimizers close to the critical coupling strength. As $a \rightarrow a^{*}$, a minimizer $u_{a}$ of (1.1) behaves like

$$
u_{a}(x) \approx \frac{\lambda}{\|Q\|_{2}\left(a^{*}-a_{k}\right)^{\frac{1}{2+p}}} Q\left(\frac{\lambda\left(x-x_{0}\right)}{\left(a^{*}-a_{k}\right)^{\frac{1}{2+p}}}\right),
$$

with $x_{0}$ a minimum of $V(x)$, and $\lambda$ the smallest of the values $\lambda_{i}$ defined in (1.10). Such an equality can, in general, hold only for a subsequence. If $x_{0}$ is unique, however, i.e., $|\mathcal{Z}|=1$, it is not necessary to go to a subsequence, and the convergence (1.12) holds for any sequence.

If the trap potential $V$ has a symmetry, e.g., $V(x)=\prod_{i=1}^{n}\left|x-x_{i}\right|^{p}$ with $p>0$ and the $x_{i}$ arranged on the vertices of a regular polygon, Theorem 2 establishes the symmetry breaking occurring in the GP minimizers. There exists an $a_{*}$, with $0<a_{*}<a^{*}$, such that for $a_{*}<a<a^{*}$, the GP functional (1.1) has (at least) $n$ different non-negative minimizers, each of which concentrates at a specific global minimum point $x_{i}$.

One can show that symmetry breaking can only occur for $a$ sufficiently large. That is, for small enough $a$, there is always a unique minimizer (up to multiplication by a constant phase), as in the case $a=0$. This can be proved using the technique employed in the proof of Theorem 2 in [3] (see also [35] and Theorem 1.1 in [31]). We note that the symmetry breaking bifurcation for ground states for nonlinear Schrödinger/Gross-Pitaevskii equations has been studied in detail in the literature, see, e.g., [19, 21, 22].

Our proof of Theorem 2 is based on precise estimates on the GP energy $e(a)$. In fact, we shall show that

$$
e(a) \approx\left(a^{*}-a\right)^{p /(2+p)} \frac{\lambda^{2}}{a^{*}} \frac{p+2}{p} \quad \text { as } a \rightarrow a^{*} .
$$


Note that the convergence in (1.12) also implies that

$$
\int_{\mathbb{R}^{2}}\left|u_{a}(x)\right|^{4} d x \approx 2\left(a^{*}-a\right)^{-2 /(2+p)} \frac{\lambda^{2}}{a^{*}} \quad \text { as } a \rightarrow a^{*}
$$

for a minimizer $u_{a}$.

The results in the present paper can be extended not only to more general trapping potentials $V$, but also to space dimensions $d$ different from 2 , if the exponent 4 in the last term in (1.1) is replaced by $p=1+\frac{4}{d}$. Previous results in 31] were restricted to the subcritical case $p<1+\frac{4}{d}$, where one studies the behavior of minimizers as $a \rightarrow \infty$. The case of a non-local nonlinearity was considered in 3 . Concentration phenomena have also been studied elsewhere in different context. For instance, there is a considerable literature on concentration phenomena of positive ground states of the elliptic equation

$$
h^{2} \Delta u(x)-V(x) u(x)+u(x)^{p}=0 \quad \text { in } \mathbb{R}^{d}
$$

as $h \rightarrow 0^{+}$, see [13, 33, 37, 7] and references therein.

In the remainder of this paper, we shall give the proof of Theorems 1 and 2

\section{Proof of Theorem 1}

In this section, we prove Theorem 1 on the threshold $a^{*}$, which can be defined as

$$
a^{*}=\sup \{a>0 \mid(1.4) \text { possesses at least one minimizer }\} \text {. }
$$

Recall that $Q$ is the unique positive radial solution of (1.5), and that $\frac{1}{2} \int_{\mathbb{R}^{2}} Q(x)^{2} d x$ is the minimum of the energy functional

$$
I(u)=\frac{\int_{\mathbb{R}^{2}}|\nabla u(x)|^{2} d x \int_{\mathbb{R}^{2}}|u(x)|^{2} d x}{\int_{\mathbb{R}^{2}}|u(x)|^{4} d x}, \text { where } u \in H^{1}\left(\mathbb{R}^{2}\right) .
$$

The function $Q$ is decreasing away from the origin, and [14, Prop. 4.1]

$$
Q(x),|\nabla Q(x)|=O\left(|x|^{-\frac{1}{2}} e^{-|x|}\right) \quad \text { as }|x| \rightarrow \infty .
$$

We first discuss the following bounds on $\|Q\|_{2}^{2}$.

Lemma 1. Let $Q$ be the unique positive radial solution of (1.5). Then we have

$$
2 \pi \leq \int_{\mathbb{R}^{2}} Q(x)^{2} d x \leq 2 \pi e \ln 2 \approx 2 \pi \times 1.88417 \cdots .
$$

Proof. The lower bound was proved in 24. To obtain the upper bound, we consider the trial functions $u_{\gamma}(x)=e^{-|x|^{\gamma / 2}}$ for $\gamma>0$. Simple calculations yield

$$
\left\|u_{\gamma}\right\|_{2}^{2}=\pi \Gamma(1+2 / \gamma)=2^{2 / \gamma}\left\|u_{\gamma}\right\|_{4}^{4}, \quad\left\|\nabla u_{\gamma}\right\|_{2}^{2}=\frac{\pi \gamma}{2},
$$

and hence

$$
\int_{\mathbb{R}^{2}} Q(x)^{2} d x \leq 2 \inf _{\gamma>0} I\left(u_{\gamma}\right)=\inf _{\gamma>0} \pi \gamma 2^{2 / \gamma}=2 \pi e \ln 2,
$$


where the infimum is attained at $\gamma=\ln 4$.

The accuracy of our upper bound in Lemma 1 can be observed from the numerical result $\|Q\|_{2}^{2}=2 \pi \times 1.86225 \cdots$ in 38 .

The following compactness result is well known, see, e.g., [1.

Lemma 2. Suppose $V \in L_{\mathrm{loc}}^{\infty}\left(\mathbb{R}^{2}\right)$ with $\lim _{|x| \rightarrow \infty} V(x)=\infty$. If $2 \leq q<\infty$, then the embedding $\mathcal{H} \hookrightarrow L^{q}\left(\mathbb{R}^{2}\right)$ is compact.

This compactness property, together with the Gagliardo-Nirenberg inequality (1.6), allows us to prove Theorem 1. We shall first show that (1.4) admits at least one minimizer provided that $0 \leq a<\|Q\|_{2}^{2}$. The proof of this fact is essentially the same as the one in [40], where the special case $V(x)=|x|^{2}$ was considered (see also [35]). We give it here for completeness.

If $u \in \mathcal{H}$ and $\|u\|_{2}^{2}=1$, then for all $0 \leq a<\|Q\|_{2}^{2}$ we observe from (1.6) and the positivity of $V$ that

$$
\begin{aligned}
E_{a}(u) & \geq\left(1-\frac{a}{\|Q\|_{2}^{2}}\right) \int_{\mathbb{R}^{2}}|\nabla u|^{2} d x+\int_{\mathbb{R}^{2}} V(x)|u(x)|^{2} d x \\
& \geq\left(1-\frac{a}{\|Q\|_{2}^{2}}\right) \int_{\mathbb{R}^{2}}|\nabla u|^{2} d x,
\end{aligned}
$$

which implies that $E_{a}(u)$ is bounded from below. Let $\left\{u_{n}\right\} \in \mathcal{H}$ be a sequence satisfying $\left\|u_{n}\right\|_{2}=1$ and $\lim _{n \rightarrow \infty} E_{a}\left(u_{n}\right)=e(a)$. Because of (2.5), we see that both $\int_{\mathbb{R}^{2}}\left|\nabla u_{n}(x)\right|^{2} d x$ and $\int_{\mathbb{R}^{2}} V(x)\left|u_{n}(x)\right|^{2} d x$ are uniformly bounded in $n$. By the compactness of Lemma 2 we can extract a subsequence such that

$$
u_{n} \rightarrow u \text { weakly in } \mathcal{H}, \quad u_{n} \rightarrow u \text { strongly in } L^{q}\left(\mathbb{R}^{2}\right), \quad 2 \leq q<\infty
$$

for some $u \in \mathcal{H}$. We conclude that $\int_{\mathbb{R}^{2}}|u(x)|^{2} d x=1$ and $E_{a}(u)=e(a)$, by weak lower semicontinuity. This implies the existence of minimizers for any $0 \leq a<\|Q\|_{2}^{2}$.

To prove that there is no minimizer for (1.4) as soon as $a \geq\|Q\|_{2}^{2}$, we proceed as follows. Choose a non-negative $\varphi \in C_{0}^{\infty}\left(\mathbb{R}^{2}\right)$ such that $\varphi(x)=1$ for $|x| \leq 1$. For $x_{0} \in \mathbb{R}^{2}, \tau>0$ and $R>0$, let

$$
u(x)=A_{R, \tau} \frac{\tau}{\|Q\|_{2}} \varphi\left(\left(x-x_{0}\right) / R\right) Q\left(\tau\left(x-x_{0}\right)\right),
$$

where $A_{R, \tau}>0$ is chosen so that $\int_{\mathbb{R}^{2}} u(x)^{2} d x=1$. By scaling, $A_{R, \tau}$ depends only on the product $R \tau$, and we have $\lim _{\tau \rightarrow \infty} A_{R, \tau}=1$. In fact,

$$
\frac{1}{A_{R, \tau}^{2}}=\frac{1}{\|Q\|_{2}^{2}} \int_{\mathbb{R}^{2}} Q(x)^{2} \varphi(x /(\tau R))^{2} d x=1+O\left((R \tau)^{-\infty}\right) \quad \text { as } R \tau \rightarrow \infty
$$

because of the exponential decay of $Q$ in Eq. (2.3). Here we use the notation $f(t)=O\left(t^{-\infty}\right)$ for a function $f$ satisfying $\lim _{t \rightarrow \infty}|f(t)| t^{s}=0$ for all $s>0$. In the following, we could set $R=1$, for instance.

Using the exponential decay of both $Q$ and $\nabla Q$, we also have

$$
\begin{aligned}
& \int_{\mathbb{R}^{2}}|\nabla u(x)|^{2} d x-\frac{a}{2} \int_{\mathbb{R}^{2}} u(x)^{4} d x \\
& =\frac{\tau^{2}}{\|Q\|_{2}^{2}}\left[\int_{\mathbb{R}^{2}}|\nabla Q(x)|^{2} d x-\frac{a}{2\|Q\|_{2}^{2}} \int_{\mathbb{R}^{2}} Q(x)^{4} d x+O\left((R \tau)^{-\infty}\right)\right] \quad \text { as } \quad R \tau \rightarrow \infty .
\end{aligned}
$$


Since $\|\nabla Q\|_{2}^{2}=\frac{1}{2}\|Q\|_{4}^{4}$, we further have

$$
(\underline{2.8)})=\frac{\tau^{2}}{2\|Q\|_{2}^{2}}\left[\left(1-\frac{a}{\|Q\|_{2}^{2}}\right) \int_{\mathbb{R}^{2}} Q(x)^{4} d x+O\left((R \tau)^{-\infty}\right)\right] \quad \text { as } \quad R \tau \rightarrow \infty .
$$

On the other hand, since the function $x \mapsto V(x) \varphi\left(\left(x-x_{0}\right) / R\right)^{2}$ is bounded and has compact support, the convergence

$$
\lim _{\tau \rightarrow \infty} \int_{R^{2}} V(x) u(x)^{2} d x=V\left(x_{0}\right)
$$

holds for almost every $x_{0} \in \mathbb{R}^{2}[26$.

For $a>\|Q\|_{2}^{2}$, it follows from (2.9) and (2.10) that

$$
e(a) \leq \lim _{\tau \rightarrow \infty} E_{a}(u)=-\infty .
$$

This implies that for any $a>\|Q\|_{2}^{2}, e(a)$ is unbounded from below, and the non-existence of minimizers is therefore proved. In the case $a=\|Q\|_{2}^{2}$, (2.9) and (2.10) show in combination that $e(a) \leq V\left(x_{0}\right)$. This holds for almost every $x_{0}$; taking the infimum over $x_{0}$ yields $e(a) \leq 0$. There is, in fact, equality in this case, as (2.5) shows. Suppose now that there exists a minimizer $u$ at $a=\|Q\|_{2}^{2}$. As pointed out in the Introduction, we can assume $u$ to be non-negative. We would then have

$$
\int_{\mathbb{R}^{2}} V(x)|u(x)|^{2} d x=\inf _{x \in \mathbb{R}^{2}} V(x)=0
$$

and

$$
\int_{\mathbb{R}^{2}}|\nabla u(x)|^{2}=\frac{1}{2} \int_{\mathbb{R}^{2}}|u(x)|^{4} d x .
$$

This is a contradiction, since for the first equality $u$ would have to have compact support, while for the second one it has to be equal to (a translation of) $Q$.

This completes the proof of the first part of Theorem 1 To prove the stated properties of the GP energy $e(a)$, note that (2.5) implies that $e(a)>0$ for $a<a^{*}=\|Q\|_{2}^{2}$. We have already shown that $e\left(a^{*}\right)=0$ and $e(a)=-\infty$ for $a>a^{*}$, hence it remains to show that $\lim _{a \rightarrow a^{*}} e(a)=0$. This follows easily from (2.9) and (2.10), by first taking $a \rightarrow a^{*}$, followed

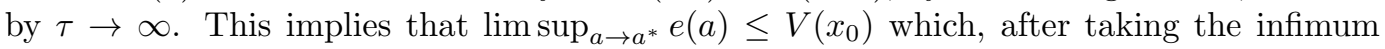
over $x_{0}$, yields the result.

\section{Proof of Theorem 2}

We shall now restrict our attention to trap potentials $V$ satisfying (1.9). We have already shown in Theorem 1 that $e(a) \searrow 0$ as $a \nearrow a^{*}$, where $e(a)$ is the GP energy defined in (1.4). In the following we shall derive refined estimates on $e(a)$.

Lemma 3. Suppose $V$ satisfies (1.9). Then there exist two positive constants $m<M$, independent of a, such that

$$
m\left(a^{*}-a\right)^{\frac{p}{p+2}} \leq e(a) \leq M\left(a^{*}-a\right)^{\frac{p}{p+2}} \text { for } 0 \leq a \leq a^{*},
$$

where $p>0$ is defined before (1.10). 
Proof. Since $e(a)$ is decreasing and uniformly bounded for $0 \leq a \leq a^{*}$, it suffices to consider the case when $a$ is close to $a^{*}$. We start with the lower bound. From (1.6) we infer that, for any $\gamma>0$ and $u \in \mathcal{H}$ with $\|u\|_{2}=1$,

$$
\begin{aligned}
E_{a}(u) & \geq \int_{\mathbb{R}^{2}} V(x)|u(x)|^{2} d x+\frac{a^{*}-a}{2} \int_{\mathbb{R}^{2}}|u(x)|^{4} d x \\
& =\gamma+\int_{\mathbb{R}^{2}}\left[(V(x)-\gamma)|u(x)|^{2}+\frac{a^{*}-a}{2}|u(x)|^{4}\right] d x \\
& \geq \gamma-\frac{1}{2\left(a^{*}-a\right)} \int_{\mathbb{R}^{2}}[\gamma-V(x)]_{+}^{2} d x,
\end{aligned}
$$

where $[\cdot]_{+}=\max \{0, \cdot\}$ denotes the positive part. For small enough $\gamma$, the set

$$
\left\{x \in \mathbb{R}^{2}: V(x) \leq \gamma\right\}
$$

is contained in the disjoint union of $n$ balls of radius at most $K \gamma^{1 / p}$, centered at the minima $x_{i}$, for a suitable constant $K>0$. Moreover, $V(x) \geq\left(\left|x-x_{i}\right| / K\right)^{p}$ on these balls. Hence

$$
\int_{\mathbb{R}^{2}}[\gamma-V(x)]_{+}^{2} d x \leq n \int_{\mathbb{R}^{2}}\left[\gamma-(|x| / K)^{p}\right]_{+}^{2} d x=C \gamma^{2+2 / p}
$$

with $C=n K^{2} \pi p^{2} /[(p+1)(p+2)]$. The lower bound in (3.1) therefore follows from (3.2) by taking $\gamma$ to be equal to $\left[p\left(a^{*}-a\right) /(C(1+p))\right]^{p /(2+p)}$.

Next we shall prove the upper bound in (3.1). We proceed similarly to the proof of Theorem 11, and use a trial function of the form (2.6), with $0 \leq \varphi \leq 1$. Recall the definition of $\mathcal{Z}$ in (1.11), and pick $x_{0} \in \mathcal{Z}$. We choose $R$ small enough so that

$$
V(x) \leq C\left|x-x_{0}\right|^{p} \quad \text { for }\left|x-x_{0}\right| \leq R,
$$

in which case we have

$$
\int_{\mathbb{R}^{2}} V(x) u(x)^{2} d x \leq C \tau^{-p} A_{R, \tau}^{2} \int_{\mathbb{R}^{2}}|x|^{p} Q(x)^{2} d x .
$$

From the estimates (2.7)-(2.9) we thus conclude that, for large $\tau$,

$$
e(a) \leq \frac{\tau^{2}}{2\left(a^{*}\right)^{2}}\left(a^{*}-a\right) \int_{\mathbb{R}^{2}} Q(x)^{4} d x+C \tau^{-p} \int_{\mathbb{R}^{2}}|x|^{p} Q(x)^{2} d x+O\left(\tau^{-\infty}\right) .
$$

By taking $\tau=\left(a^{*}-a\right)^{-\frac{1}{p+2}}$, we arrive at the desired upper bound. This completes the proof of the lemma.

Let $u_{a}$ be a minimizer of (1.1). The following bound on the $L^{4}\left(\mathbb{R}^{2}\right)$ norm of $u_{a}$ is a simple consequence of Lemma 3

Lemma 4. Suppose $u_{a}$ is a minimizer of (1.1) with $V$ satisfying (1.9). Then there exists a positive constant $K$, independent of a, such that

$$
0<K\left(a^{*}-a\right)^{-\frac{2}{p+2}} \leq \int_{\mathbb{R}^{2}}\left|u_{a}(x)\right|^{4} d x \leq \frac{1}{K}\left(a^{*}-a\right)^{-\frac{2}{p+2}} \quad \text { for } 0 \leq a<a^{*} .
$$


Proof. Since by (2.5) and (1.6)

$$
e(a) \geq \frac{a^{*}-a}{2} \int_{\mathbb{R}^{2}}\left|u_{a}(x)\right|^{4} d x,
$$

the upper bound in (3.3) follows immediately from Lemma 3

To prove the lower bound in (3.3), we pick a $0<b<a$ and use that

$$
e(b) \leq E_{b}\left(u_{a}\right)=e(a)+\frac{a-b}{2} \int_{\mathbb{R}^{2}}\left|u_{a}(x)\right|^{4} d x .
$$

By applying Lemma 3, the above inequality implies that there exist two positive constants $m<M$ such that for any $0<b<a<a^{*}$,

$$
\frac{1}{2} \int_{\mathbb{R}^{2}}\left|u_{a}(x)\right|^{4} d x \geq \frac{e(b)-e(a)}{a-b} \geq \frac{m\left(a^{*}-b\right)^{p /(2+p)}-M\left(a^{*}-a\right)^{p /(2+p)}}{a-b} .
$$

With $b=a-\gamma\left(a^{*}-a\right)$, we can write the right side of (3.4) as

$$
\left(a^{*}-a\right)^{-2 /(2+p)} \frac{m(1+\gamma)^{p /(2+p)}-M}{\gamma} .
$$

The last fraction is positive for $\gamma$ large enough. For $a$ close to $a^{*}$, this then gives the desired lower bound. For smaller $a$, one can simply use the fact that $\int\left|u_{a}(x)\right|^{4} d x \geq \int\left|u_{0}(x)\right|^{4} d x$ for any $0 \leq a \leq a^{*}$, which follows from concavity of $e(a)$ (or from the bounds $e(a) \leq E_{a}\left(u_{0}\right)$ and $\left.e(0) \leq E_{0}\left(u_{a}\right)\right)$. This completes the proof of the lemma.

Let $u_{a}$ be a non-negative minimizer of (1.4), and define

$$
\varepsilon:=\left(a^{*}-a\right)^{\frac{1}{2+p}}>0 .
$$

From (1.6) we conclude that

$$
e(a) \geq\left(1-\frac{a}{a^{*}}\right) \int_{\mathbb{R}^{2}}\left|\nabla u_{a}(x)\right|^{2} d x+\int_{\mathbb{R}^{2}} V(x) u_{a}(x)^{2} d x,
$$

and hence it follows from Lemma 3 that

$$
\int_{\mathbb{R}^{2}}\left|\nabla u_{a}(x)\right|^{2} d x \leq C \varepsilon^{-2} \text { and } \int_{\mathbb{R}^{2}} V(x) u_{a}(x)^{2} d x \leq C \varepsilon^{p} .
$$

For $1 \leq i \leq n$, define the $L^{2}\left(\mathbb{R}^{2}\right)$-normalized functions

$$
w_{a}^{(i)}(x):=\varepsilon u_{a}\left(\varepsilon x+x_{i}\right) .
$$

From (3.6) and Lemma 4, we have

$$
0<K \leq \int_{\mathbb{R}^{2}} w_{a}^{(i)}(x)^{4} d x \leq \frac{1}{K}, \quad \int_{\mathbb{R}^{2}}\left|\nabla w_{a}^{(i)}(x)\right|^{2} d x \leq C
$$

and also

$$
\int_{\mathbb{R}^{2}} V\left(x_{i}+\varepsilon x\right) w_{a}^{(i)}(x)^{2} d x \leq C \varepsilon^{p} .
$$


In particular, the functions $w_{a}^{(i)}$ are bounded uniformly in $H^{1}\left(\mathbb{R}^{2}\right)$.

For any $\gamma>0$, we have

$$
\int_{\left\{V(x) \geq \gamma \varepsilon^{p}\right\}} u_{a}(x)^{2} d x \leq \frac{1}{\gamma \varepsilon^{p}} \int_{\mathbb{R}^{2}} V(x) u_{a}(x)^{2} d x \leq \frac{C}{\gamma} .
$$

For $\varepsilon$ small enough, i.e., for $a$ sufficiently close to $a^{*}$, the set $\left\{x \in \mathbb{R}^{2}: V(x) \leq \gamma \varepsilon^{p}\right\}$ is contained in $n$ disjoint balls with radius at most $C \gamma^{1 / p} \varepsilon$, for some $C>0$, centered at the points $x_{i}$. We thus deduce from the above inequality that

$$
\begin{aligned}
\frac{C}{\gamma} & \geq \int_{\left\{V(x) \geq \gamma \varepsilon^{p}\right\}} u_{a}(x)^{2} d x=1-\int_{\left\{V(x) \leq \gamma \varepsilon^{p}\right\}} u_{a}(x)^{2} d x \\
& \geq 1-\sum_{i=1}^{n} \int_{\left\{\left|x-x_{i}\right| \leq C \gamma^{1 / p} \varepsilon\right\}} u_{a}(x)^{2} d x=1-\sum_{i=1}^{n} \int_{\left\{|x| \leq C \gamma^{1 / p}\right\}} w_{a}^{(i)}(x)^{2} d x .
\end{aligned}
$$

In particular,

$$
1 \geq \sum_{i=1}^{n} \int_{\left\{|x| \leq C \gamma^{1 / p}\right\}} w_{a}^{(i)}(x)^{2} d x \geq 1-\frac{C}{\gamma} .
$$

Since the functions $w_{a}^{(i)}$ are uniformly bounded in $H^{1}\left(\mathbb{R}^{2}\right)$, we can pass to a subsequence such that

$$
w_{a}^{(i)} \rightarrow w_{0}^{(i)} \text { weakly in } H^{1}\left(\mathbb{R}^{2}\right), \quad 1 \leq i \leq n,
$$

for suitable functions $w_{0}^{(i)} \in H^{1}\left(\mathbb{R}^{2}\right)$. By Lemma 2 the convergence holds strongly in $L^{q}\left(\left\{|x| \leq C \gamma^{1 / p}\right\}\right)$ for any $2 \leq q<\infty$. In particular, from (3.10) we conclude that

$$
1 \geq \sum_{i=1}^{n} \int_{\left\{|x| \leq C \gamma^{1 / p}\right\}} w_{0}^{(i)}(x)^{2} d x \geq 1-\frac{C}{\gamma} .
$$

Since this bound holds for any $\gamma>0$, we finally conclude that

$$
\sum_{i=1}^{n}\left\|w_{0}^{(i)}\right\|_{2}^{2}=1
$$

Since $u_{a}$ is a minimizer of (1.4), it satisfies the Euler-Lagrange equation

$$
-\Delta u_{a}(x)+V(x) u_{a}(x)=\mu_{a} u_{a}(x)+a u_{a}(x)^{3} \quad \text { in } \mathbb{R}^{2}
$$

for $\mu_{a} \in \mathbb{R}$ a suitable Lagrange multiplier. In fact,

$$
\mu_{a}=e(a)-\frac{a}{2} \int_{\mathbb{R}^{2}} u_{a}(x)^{4} d x .
$$

The functions $w_{a}^{(i)}$ in (3.7) are thus non-negative solutions of

$$
-\Delta w_{a}^{(i)}(x)+\varepsilon^{2} V\left(x_{i}+\varepsilon x\right) w_{a}^{(i)}(x)=\varepsilon^{2} \mu_{a} w_{a}^{(i)}(x)+a w_{a}^{(i)}(x)^{3} \quad \text { in } \quad \mathbb{R}^{2} .
$$

It follows from Lemma 4 that $\varepsilon^{2} \mu_{a}$ is uniformly bounded as $a \rightarrow a^{*}$, and strictly negative for $a$ close to $a^{*}$. By passing to a subsequence, if necessary, we can thus assume that $\varepsilon^{2} \mu_{a}$ 
converges to some number $-\beta^{2}<0$ as $a \rightarrow a^{*}$. By passing to the weak limit (3.11), we see that the non-negative functions $w_{0}^{(i)}$ satisfy

$$
-\Delta w_{0}^{(i)}(x)=-\beta^{2} w_{0}^{(i)}(x)+a^{*} w_{0}^{(i)}(x)^{3} .
$$

By the maximum principle, either $w_{0}^{(i)}=0$ identically, or otherwise $w_{0}^{(i)}>0$ for all $x \in \mathbb{R}^{2}$. In the latter case, a simple rescaling together with the uniqueness of positive solutions of (1.5) up to translations allows us to conclude that

$$
w_{0}^{(i)}(x)=\frac{\beta}{\|Q\|_{2}} Q\left(\beta\left(x-y_{i}\right)\right) \quad \text { for some } y_{i} \in \mathbb{R}^{2} .
$$

In particular, either $w_{0}^{(i)}=0$ or $\left\|w_{0}^{(i)}\right\|_{2}^{2}=1$. Because of (3.12), we see that exactly one $w_{0}^{(i)}$ is of the form (3.16), while all the others are zero.

Let $1 \leq j \leq n$ be such that $\left\|w_{0}^{(j)}\right\|_{2}=1$. From the norm preservation we conclude that $w_{a}^{(j)}$ converges to $w_{0}^{(j)}$ strongly in $L^{2}\left(\mathbb{R}^{2}\right)$ and, in fact, strongly in $L^{q}\left(\mathbb{R}^{2}\right)$ for any $2 \leq q<\infty$ because of $H^{1}\left(\mathbb{R}^{2}\right)$ boundedness. By going to a subsequence, if necessary, we can also assume that the convergence holds pointwise almost everywhere.

To complete the proof of Theorem 2, we compute

$$
\begin{aligned}
e(a)=E_{a}\left(u_{a}\right)= & \frac{1}{\varepsilon^{2}}\left[\int_{\mathbb{R}^{2}}\left|\nabla w_{a}^{(j)}(x)\right|^{2} d x-\frac{a^{*}}{2} \int_{\mathbb{R}^{2}} w_{a}^{(j)}(x)^{4} d x\right] \\
& +\frac{\varepsilon^{p}}{2} \int_{\mathbb{R}^{2}} w_{a}^{(j)}(x)^{4} d x+\int_{\mathbb{R}^{2}} V\left(x_{j}+\varepsilon x\right) w_{a}^{(j)}(x)^{2} d x .
\end{aligned}
$$

The term in square brackets is non-negative and can be dropped for a lower bound. The $L^{4}\left(\mathbb{R}^{2}\right)$ norm of $w_{a}^{(j)}$ converges to the one of $w_{0}^{(j)}$, and from Fatou's Lemma it follows that

$$
\liminf _{\varepsilon \rightarrow 0} \varepsilon^{-p} \int_{\mathbb{R}^{2}} V\left(x_{j}+\varepsilon x\right) w_{a}^{(j)}(x)^{2} d x \geq \kappa_{j} \int_{\mathbb{R}^{2}}|x|^{p} w_{0}^{(j)}(x)^{2} d x,
$$

where $\kappa_{j}=\lim _{x \rightarrow x_{j}} V(x)\left|x-x_{j}\right|^{-p} \in(0, \infty]$. Moreover,

$$
\int_{\mathbb{R}^{2}}|x|^{p} w_{0}^{(j)}(x)^{2} d x=\frac{1}{\beta^{p}\|Q\|_{2}^{2}} \int_{\mathbb{R}^{2}}\left|x+y_{j}\right|^{p} Q(x)^{2} d x \geq \frac{1}{\beta^{p}\|Q\|_{2}^{2}} \int_{\mathbb{R}^{2}}|x|^{p} Q(x)^{2} d x
$$

since $Q$ is a radial decreasing function. In particular,

$$
\liminf _{a \rightarrow a^{*}} \frac{e(a)}{\left(a^{*}-a\right)^{p /(2+p)}} \geq \frac{1}{2}\left\|w_{0}\right\|_{4}^{4}+\kappa_{j} \int_{\mathbb{R}^{2}}|x|^{p} w_{0}^{(j)}(x)^{2} d x \geq \frac{2}{a^{*}}\left(\frac{\beta^{2}}{2}+\lambda_{j}^{2+p} \frac{1}{p \beta^{p}}\right),
$$

where $\lambda_{j}$ is defined in (1.10), and we have used that $\|Q\|_{4}^{4}=2\|Q\|_{2}^{2}=2 a^{*}$ (which follows from the fact that $Q$ satisfies Eq. (1.5) and equality in (1.6)). Taking the infimum over $\beta>0$ (which is achieved for $\beta=\lambda_{j}$ ) yields

$$
\liminf _{a \rightarrow a^{*}} \frac{e(a)}{\left(a^{*}-a\right)^{p /(2+p)}} \geq \frac{\lambda^{2}}{a^{*}} \frac{p+2}{p},
$$

where $\lambda=\min _{j} \lambda_{j}$, as before. 
The limit in (3.19) actually exists, and is equal to the right side. To see this, one simply takes

$$
u(x)=\frac{\beta}{\varepsilon\|Q\|_{2}} Q\left(\beta \frac{x-x_{j}}{\varepsilon}\right)
$$

as a trial function for $E_{a}$, and minimizes over $1 \leq j \leq n$ and $\beta>0$. The result is that

$$
\lim _{a \rightarrow a^{*}} \frac{e(a)}{\left(a^{*}-a\right)^{p /(2+p)}}=\frac{\lambda^{2}}{a^{*}} \frac{p+2}{p} .
$$

From the equality (3.20) we can draw several conclusions. First, the $j$ defined above is such that $\lambda_{j}=\lambda$, i.e., $x_{j} \in \mathcal{Z}$. Second, $\beta$ is unique (i.e., independent of the choice of the subsequence) and equal to the expression minimizing (3.18), i.e., $\beta=\lambda$. Finally, $y_{j}=0$, since the inequality (3.17) is strict for $y_{j} \neq 0$. We have thus shown that

$$
w_{a}^{(j)}(x)=\varepsilon u_{a}\left(\varepsilon x+x_{j}\right) \rightarrow \frac{\lambda}{\|Q\|_{2}} Q(\lambda x) \text { as } a \rightarrow a^{*},
$$

with $x_{j} \in \mathcal{Z}$. This completes the proof of Theorem 2 .

\section{References}

[1] R.A. Adams, J.J.F. Fournier, Sobolev spaces, $2^{\text {nd }}$ ed., Academic Press (2003).

[2] M.H. Anderson, J.R. Ensher, M.R. Matthews, C.E. Wieman, E.A. Cornell, Observation of Bose-Einstein condensation in a dilute atomic vapor, Science 269, 198-201 (1995).

[3] W.H. Aschbacher, J. Fröhlich, G.M. Graf, K. Schnee, M. Troyer, Symmetry breaking regime in the nonlinear Hartree equation, J. Math. Phys. 43, 3879-3891 (2002).

[4] I. Bloch, J. Dalibard, and W. Zwerger, Many-body physics with ultracold gases, Rev. Mod. Phys. 80, 885 (2008).

[5] C.C. Bradley, C.A. Sackett, J.J. Tollett, R.G. Hulet, Evidence of Bose-Einstein Condensation in an Atomic Gas with Attractive Interactions, Phys. Rev. Lett. 75, 1687 (1995). Erratum Phys. Rev. Lett. 79, 1170 (1997).

[6] C.C. Bradley, C.A. Sackett, R.G. Hulet, Bose-Einstein Condensation of Lithium: Observation of Limited Condensate Number, Phys. Rev. Lett. 78, 985 (1997).

[7] J. Byeon, Z.Q. Wang, Standing waves with a critical frequency for nonlinear Schrödinger equations, Arch. Ration. Mech. Anal. 165, 295-316 (2002).

[8] T. Cazenave, Semilinear Schrödinger equations, Courant Lecture Notes in Mathematics 10, Courant Institute of Mathematical Science/AMS, New York (2003).

[9] N.R. Cooper, Rapidly rotating atomic gases, Adv. Phys. 57, 539-616 (2008).

[10] F. Dalfovo, S. Giorgini, L.P. Pitaevskii, S. Stringari, Theory of Bose-Einstein condensation in trapped gases, Rev. Mod. Phys. 71, 463-512 (1999). 
[11] K.B. Davis, M.O. Mewes, M.R. Andrews, N.J. van Druten, D.S. Durfee, D.M. Kurn, W. Ketterle, Bose-Einstein condensation in a gas of sodium atoms, Phys. Rev. Lett. 75, 3969-3973 (1995).

[12] A.L. Fetter, Rotating trapped Bose-Einstein condensates, Rev. Mod. Phys. 81, 647 (2009).

[13] A. Floer, A. Weinstein, Nonspreading wave packets for the cubic Schrödinger equation with a bounded potential, J. Funct. Anal. 69, 397-408 (1986).

[14] B. Gidas, W.M. Ni, L. Nirenberg, Symmetry of positive solutions of nonlinear elliptic equations in $\mathbb{R}^{n}$, Mathematical analysis and applications Part A, Adv. in Math. Suppl. Stud. vol. 7, Academic Press, New York, 369-402 (1981).

[15] E.P. Gross, Structure of a quantized vortex in boson systems, Nuovo Cimento 20, 454466 (1961).

[16] E.P. Gross, Hydrodynamics of a superfluid condensate, J. Math. Phys. 4, 195-207 (1963).

[17] Y.J. Guo, X.Y. Zeng and H.S. Zhou, Energy estimates and symmetry breaking in attractive Bose-Einstein condensates with ring-shaped potentials, preprint, (2013).

[18] C. Huepe, S. Metens, G. Dewel, P. Borckmans, M.E. Brachet, Decay rates in attractive Bose-Einstein condensates, Phys. Rev. Lett. 82, 1616-1619 (1999).

[19] R.K. Jackson and M.I. Weinstein, Geometric analysis of bifurcation and symmetry breaking in a Gross-Pitaevskii equation, J. Stat. Phys. 116, 881-905 (2004).

[20] Y. Kagan, A.E. Muryshev, G.V. Shlyapnikov, Collapse and Bose-Einstein condensation in a trapped Bose gas with negative scattering length, Phys. Rev. Lett. 81, 933-937 (1998).

[21] E.W. Kirr, P.G. Kevrekidis, E. Shlizerman, M.I. Weinstein, Symmetry-breaking bifurcation in nonlinear Schrödinger/Gross-Pitaevskii equations, SIAM J. Math. Anal. 40, 566-604 (2008).

$[22]$ E. W. Kirr, P. G. Kevrekidis and D. E. Pelinovsky, Symmetry-breaking bifurcation in the nonlinear Schrödinger equation with symmetric potentials, Comm. Math. Phys. 308, no. 3, 795-844 (2011).

[23] M.K. Kwong, Uniqueness of positive solutions of $\Delta u-u+u^{p}=0$ in $\mathbb{R}^{N}$, Arch. Rational Mech. Anal. 105, 243-266 (1989).

[24] H.A. Levine, An estimate for the best constant in a Sobolev inequality involving three integral norms, Ann. Math. Pura Appl. 124, 181-197 (1980).

[25] Y. Li, W.M. Ni, Radial symmetry of positive solutions of nonlinear elliptic equations in $\mathbb{R}^{n}$, Comm. Partial Differential Equations 18, 1043-1054 (1993).

[26] E.H. Lieb, M. Loss, Analysis, Graduate Studies in Mathematics 14, Amer. Math. Soc., Providence, RI, second edition (2001). 
[27] E.H. Lieb, R. Seiringer, Proof of Bose-Einstein condensation for dilute trapped gases, Phys. Rev. Lett. 88, 170409-1-4 (2002).

[28] E.H. Lieb, R. Seiringer, J.P. Solovej, J. Yngvason, The mathematics of the Bose gas and its condensation, Oberwolfach Seminars 34, Birkhäuser Verlag, Basel (2005).

[29] E.H. Lieb, R. Seiringer, J. Yngvason, Bosons in a trap: A rigorous derivation of the Gross-Pitaevskii energy functional, Phys. Rev. A 61, 043602-1-13 (2000).

[30] E.H. Lieb, R. Seiringer, J. Yngvason, A rigorous derivation of the Gross-Pitaevskii energy functional for a two-dimensional Bose gas, Commun. Math. Phys. 224, 17-31 (2001).

[31] M. Maeda, On the symmetry of the ground states of nonlinear Schrödinger equation with potential, Adv. Nonlinear Stud. 10, 895-925 (2010).

[32] K. McLeod, J. Serrin, Uniqueness of positive radial solutions of $\Delta u+f(u)=0$ in $\mathbb{R}^{n}$, Arch. Rational Mech. Anal. 99, 115-145 (1987).

[33] Y.-G. Oh, On positive multi-lump bound states of nonlinear Schrödinger equations under multiple well potential, Comm. Math. Phys. 131, 223-253 (1990).

[34] L.P. Pitaevskii, Vortex lines in an imperfect Bose gas, Sov. Phys. JETP. 13, 451-454 (1961).

[35] H.A. Rose and M.I. Weinstein, On the bound states of the nonlinear Schrödinger equation with a linear potential, Physica D 30, 207-218 (1988).

[36] C.A. Sackett, H.T.C. Stoof, R.G. Hulet, Growth and Collapse of a Bose-Einstein Condensate with Attractive Interactions, Phys. Rev. Lett. 80, 2031 (1998).

[37] X.F. Wang, On concentration of positive bound states of nonlinear Schrödinger equations, Comm. Math. Phys. 153, 229-244 (1993).

[38] M.I. Weinstein, Nonlinear Schrödinger equations and sharp interpolations estimates, Comm. Math. Phys. 87, 567-576 (1983).

[39] V. I. Yukalov and E. P. Yukalova, Optimal trap shape for a Bose gas with attractive interactions, Phys. Rev. A 72, 063611 (2005).

[40] J. Zhang, Stability of attractive Bose-Einstein condensates, J. Statist. Phys. 101, 731746 (2000). 